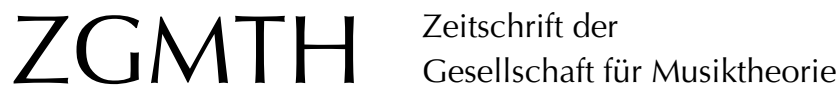

Kaiser, Ulrich (2007): Was ist ein musikalisches Modell? ZGMTH 4/3, 275-289. https://doi.org/10.31751/261

\section{(C) 2007 Ulrich Kaiser}

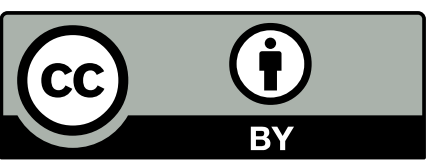

Dieser Text erscheint im Open Access und ist lizenziert unter einer Creative Commons Namensnennung 4.0 International Lizenz.

This is an open access article licensed under a

Creative Commons Attribution 4.0 International License.

veröffentlicht / first published: 01/07/2007 zuletzt geändert / last updated: 07/10/2009 


\title{
Was ist ein musikalisches Modell?
}

\author{
Ulrich Kaiser
}

Die Schriften von Carl Dahlhaus haben seit den späten 60er Jahren des 20. Jahrhunderts nicht nur den musikwissenschaftlichen, sondern auch den musiktheoretischen Diskurs maßgeblich beeinflusst. Insbesondere Dahlhaus' Forschung zu 'Satztypen und -formeln des 15. und 16. Jahrhunderts ¿ürfte die Entwicklung des Ausbildungsfachs Musiktheorie in Deutschland maßgeblich beeinflusst haben, denn Satzmodelle erlauben ein Verständnis musikalischer Strukturen jenseits von Funktionstheorie- und Ursatzdogmatik. Das nicht selten anzutreffende Herauslösen von Satzmodellen aus kompositorischen Kontexten ist jedoch nicht weniger dogmatisch. Der folgende Beitrag stellt den Versuch dar, Denkansätze aus der Informatik und Systemtheorie auf die musikalische Analyse zu übertragen und das Analysieren nach Modellen im musiktheoretischen Kontext wissenschaftstheoretisch zu fundieren.

In der Ausgabe 4/1-2 der Zeitschrift der Gesellschaft für Musiktheorie finden sich in den Beiträgen von Oliver Schwab-Felisch ${ }^{1}$ und Hans-Ulrich Fuß ${ }^{2}$ in Verbindung mit Satzmodellen die Worte sabstraktı und ıInstanz` bzw. `Instantiierung` (im Folgenden `Instanzierung`). Beide Worte werden in der Objektorientierten Programmierung (OOP) als Fachbegriffe gebraucht, mit ihnen verbindet sich heute ein spezifisches Denken bzw. umfassendes theoretisches Konzept. Obgleich dieses bereits in anderen Wissenschaftsgebieten erfolgreich adaptiert wird, sind Bemühungen, zumindest Teile des Konzepts auch für die musikalische Analyse fruchtbar zu machen, noch nicht üblich. Aus diesem Grunde wird hier der Versuch unternommen, das Denken in Klassen und Instanzen anhand des Themas ımusikalische Modelle` zu erläutern und mögliche Bedeutungen des Ansatzes für den wissenschaftlichen Diskurs des Fachs Musiktheorie aufzuzeigen. ${ }^{3}$

1 »Als abstrakte Komplexe werden Satzmodelle in ihren jeweiligen Anwendungszusammenhängen nicht zitiert, sondern instantiiert. Die Instantiierung besteht in der Bestimmung von Momenten wie Tonart, Metrum oder Rhythmus, Momenten also, die keine konstitutiven Bestandteile des jeweiligen Modellbegriffs sind, aber notwendig auf irgendeine Weise ausgeprägt sein müssen, wenn ein Satzmodell notiert oder zu Gehör gebracht werden soll.« (Schwab-Felisch 2007)

2 »Satzmodelle sind kein austauschbares Spielmaterial. Selbst für das schlichteste Klavierlied, den einfachsten Choral gilt, dass die Schemata streng genommen nicht wiederholt, sondern jedes Mal auf andere Weise instantiiert werden. Der Typus eines Satzmodells ist ein Konstrukt, will sagen eine ideale reine Form, der die komponierten Einzelfälle mehr oder minder entsprechen.« (Fuß 2007)

3 Dieser Beitrag ist eine Zusammenfassung, Konkretisierung und Weiterentwicklung der methodologischen Grundlagen meiner Untersuchungen zum jungen Mozart (Kaiser 2007). An dieser Stelle möchte ich es nicht versäumen, meinem Coach in Sachen Computerprogrammierung und Kollegen Andreas Helmberger herzlich dafür zu danken, dass er das Entstehen dieses Aufsatzes mit wertvollen Verbesserungsvorschlägen begleitet hat. 
Der Begriff `Klasse ist in der Objektorientierung ein abstrakter Oberbegriff, der die gemeinsame Struktur und das gemeinsame Verhalten von Objekten beschreibt. Klassen sind Abstraktionen von Objekten, Objekte wiederum repräsentieren Instanzen ${ }^{4}$ (Exemplare) ihrer zugehörigen Klasse. ${ }^{5}$ Verschiedene Klassen ermöglichen die Modellierung eines abgegrenzten Systems. Wenn unter `Klasse ein musikalisches Modell (z.B. eine Quintfallsequenz) und unter ১Objekt eine spezifische musikalische Wendung (z. B. bei Bach oder Händel) verstanden wird, lässt sich der Beginn dieses Absatzes wie folgt formulieren: Der Begriff `Quintfallsequenz` ist in der musikalischen Analyse ein abstrakter Oberbegriff, der die gemeinsame Struktur und das gemeinsame Verhalten von spezifischen musikalischen Wendungen z. B. im Werk von Bach oder Händel beschreibt.

Einen wichtigen Bestandteil der Objektorientierung bilden sogenannte sabstrakte Klassen . Die Bezeichnung sabstrakt irritiert auf den ersten Blick, weil im Vorangegangenen bereits die Klasse als abstrakter Oberbegriff für Objekte definiert worden ist. Der Hauptunterschied zwischen einer `Klasse letztere keine Objekte erzeugen kann bzw. sich nicht instanzieren lässt. Nehmen wir an, für die musikalische Analyse wäre eine abstrakte Klasse `Modell tonaler Musikı zu konstruieren, dann könnte diese Klasse die Deklarationen z. B. einer strukturellen Oberstimme, einer Harmoniestruktur etc. enthalten, also Deklarationen von Eigenschaften, die sich üblicher Weise an Modellen tonaler Musik beobachten lassen. Die abstrakte Klasse `Modell tonaler Musikı wird jedoch nur dann Gültigkeit für verschiedene ModellKlassen beanspruchen dürfen, wenn die in ihr vorgenommenen Deklarationen abstrakt, das heißt nicht als spezifische Eigenschaften definiert werden, da verschiedene Modelle verschiedene Oberstimmen-, Bassstimmen- und Harmoniestrukturen etc. aufweisen. Modell-Klassen wie `Quintfallsequenz` oder `Parallelismus` könnten wiederum die von der abstrakten >Modellklasse tonaler Musikı deklarierten Eigenschaften ıerbenı, was bedeutet, dass eine Verpflichtung bestände, die abstrakt deklarierten Eigenschaften zu implementieren und mit spezifischen Werten zu belegen bzw. zu überschreiben. ${ }^{6}$ Abb. 1 veranschaulicht den Sachverhalt durch ein Klassendiagramm: ${ }^{7}$

4 Um Missverständnissen vorzubeugen sei darauf hingewiesen, dass Instanzen in der Objektorientierung - im Unterschied z. B. zu Instanzen in der Rechtswissenschaft - kein hierarchisches Verhältnis untereinander aufweisen, sondern voneinander vollständig unabhängig sind. Subordinationen bestehen lediglich zwischen Instanzen und ihren zugehörigen Klassen sowie gegebenenfalls zwischen verschiedenen Klassen.

5 Klassen in der Objektorientierung sind mit den hierarchischen Klassen der biologischen Systematik vergleichbar. Die Klasse steht hier zwischen `Stamm` und `Ordnung`, wobei Klassen zu Überklassen zusammengefasst und in Unterklassen aufgeteilt werden können.

6 In diesem Aufsatz werden die in der Programmierung bekannten Begriffe sEigenschaft ‘ und `Verhalten auch für den Bereich der musikalischen Analyse verwendet und inhaltlich differenziert. Gedacht wurde dabei allerdings nicht an Attribute im Sinne klassischer Programmiersprachen, die mit Speicherorten gleichzusetzen sind. Vielmehr wird der Begriff Eigenschaft im Sinne des Konzepts der Common Language Infrastructure (CLI) verstanden, nach der Eigenschaften durch Methoden realisiert sowie abstrakt bzw. virtuell deklariert und überschrieben werden können. Darüber hinaus sind die Begriffe `Eigenschaft‘, >Attribut` etc. Kategorien der Epistemologie und Gegenstand umfänglicher Diskurse in der Philosophie. Eine Erörterung der speziellen Bedeutungen dieser Begriffe in anderen Fachgebieten ist im Rahmen dieses Aufsatzes nicht möglich. 


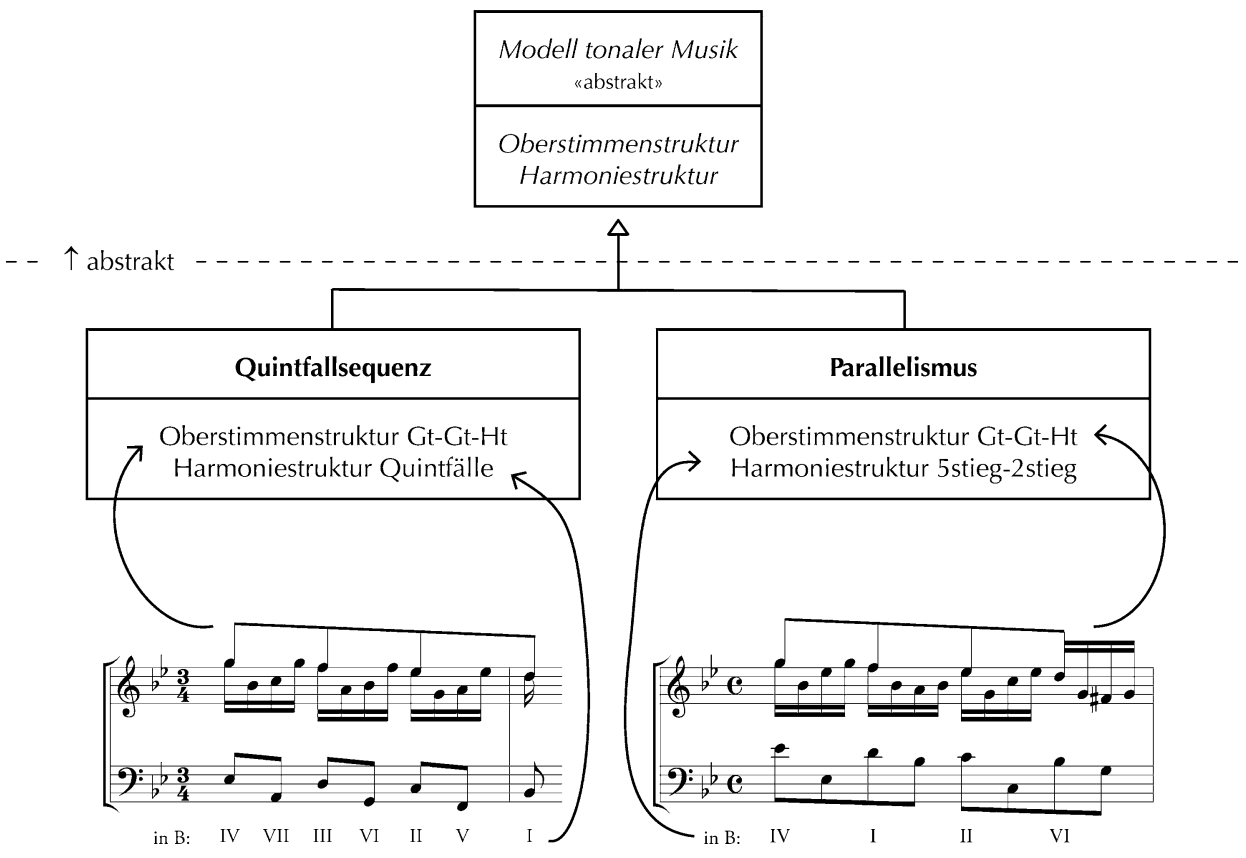

Abb. 1: Klassendiagramm für die Modell-Klassen `Quintfallsequenz` und `Parallelismus`

Beide Modell-Klassen `Quintfallsequenz` und `Parallelismus` überschreiben die Eigenschaft Oberstimmenstruktur der abstrakten Klasse >Modell tonaler Musikı auf gleiche Weise (Oberstimmenstruktur Ganzton-Ganzton-Halbton), während die Eigenschaft Harmoniestruktur auf unterschiedliche Weise implementiert wird (zum einen Quintfälle, zum anderen eine sequenzielle Folge von Quint- und Sekundstiegen). Darüber hinaus wäre es in den abgeleiteten Klassen möglich, weitere Eigenschaften zu definieren, die nicht auf Vererbung beruhen.

Zu einer Modell-Klasse (oder vereinfacht: zu einem Modell) tonaler Musik werden die genannten sowie weitere Parameter gezählt, nicht jedoch individuelle Merkmale des konkreten kompositorischen Kontextes wie z.B. die Tonart B-Dur eines spezifischen

7 Die in den Abbildungen gezeigten Klassendiagramme folgen den Standards der >Unified Modeling Language $(\mathrm{UML})$. In der 2005 offiziell freigegebenen Version 2 werden Klassen durch Rechtecke dargestellt, die je nach Erfordernissen in zwei bis drei untereinander liegende Abschnitte aufgeteilt werden können. Im obersten Abschnitt steht immer der Klassenname (fett), im Abschnitt darunter erscheinen entweder die Attribute (Datenbeschreibungen) oder das Verhalten (Operationen). Werden drei Abschnitte benötigt, befindet sich der Abschnitt der Datenbeschreibungen über dem der Operationen. Namen abstrakter Klassen und Schnittstellen werden kursiv geschrieben (und fakultativ durch den Zusatz sabstraktı bzw. ıSchnittstelle` gekennzeichnet), ebenso in abstrakten Klassen abstrakt deklarierte Verhalten oder Attribute. Durchgezogene Linien mit hohlem Pfeilkopf zeigen eine Generalisierung an, d. h. Klassen, von denen die Linie ausgeht, sspezialisieren sierung bzw. ein Klasse, auf die der Pfeil verweist. Die in Abb. 4 verwendeten gestrichelten Linien mit hohlem Pfeilkopf kennzeichnen die Verpflichtung einer Schnittstellenrealisierung. 
Werkes. Deswegen lässt sich ein Modell (z. B. ein Parallelismus) in den Werken von Schubert, Mozart oder Bach beobachten (bzw. instanzieren) und zwar unabhängig von Tonart bzw. Taktart der jeweiligen Komposition. Für eine konkrete Instanz sind dabei alle deklarierten Eigenschaften eines Modells - also auch Oberstimmen- und Harmoniestrukturen - mit Werten zu belegen (z. B. ' g-f-es-d ı als Wert der Oberstimmenstruktur Gt-Gt-Ht). ${ }^{8}$ Abb. 2 veranschaulicht eine Klassenhierarchie sowie die Instanzierung einer Modell-Klasse in unterschiedlichen tonalen Kontexten (B-Dur und F-Dur).

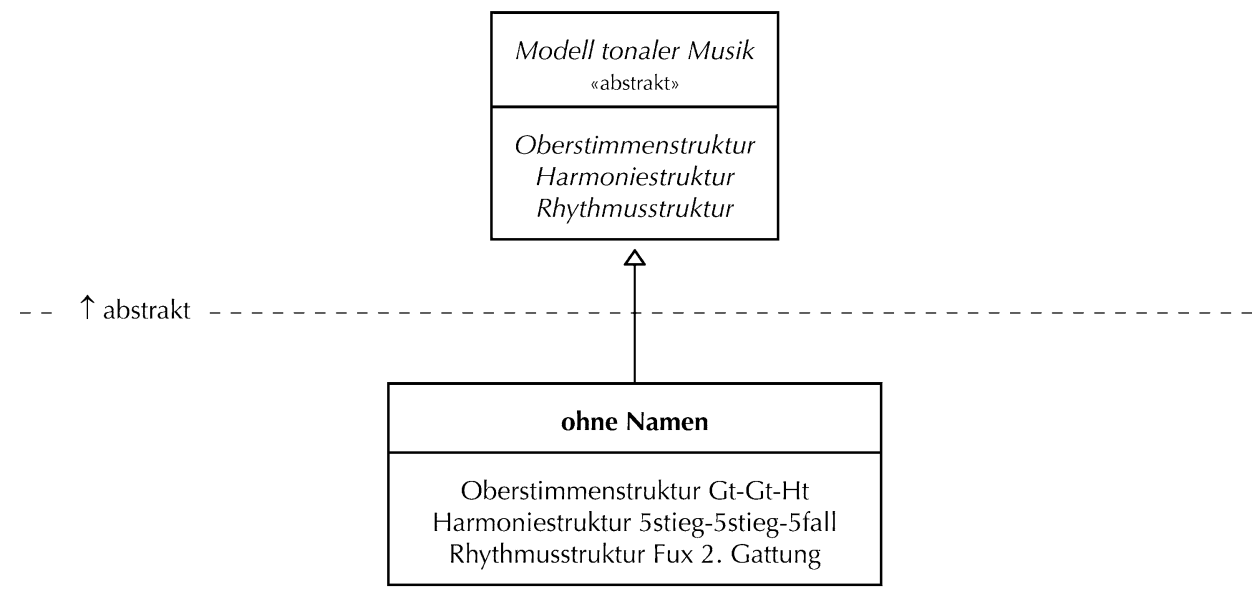

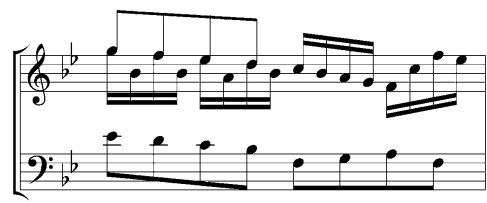

in B: IV I $\quad \mathrm{V} \quad \mathrm{I}$

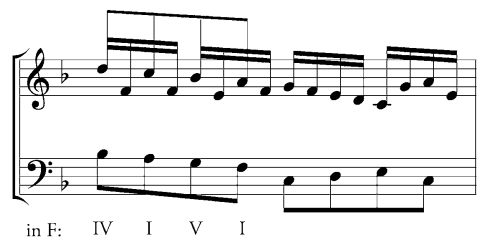

in F: IV I $\quad \mathrm{V} \quad \mathrm{I}$

Abb. 2: Instanzierung einer Klasse in unterschiedlichen tonalen Kontexten

Abb. 2 macht zudem deutlich, dass sich in komponierter Musik Instanzen von ModellKlassen aufzeigen lassen, die zwar durch charakteristische Eigenschaften ausgewiesen sind, für die jedoch kein Begriff existiert (angesichts der wenigen etablierten Fachbegriffe im Rahmen wissenschaftlichen Analysierens wahrscheinlich der Regelfall). In meiner Arbeit Die Notenbücher der Mozarts habe ich die abgebildete Modell-Klasse als

8 Bei der Wertzuweisung wurde nicht an öffentliche Speicherorte gedacht, deren Inhalt sich nach der Zuweisung verändern lässt. Vielmehr erfolgt eine Belegung mit konkreten Werten ausschließlich bei der Instanzierung von Modell-Klassen, wobei die zugewiesenen Werte nach der Instanzierung nicht mehr veränderbar sind. Die Unveränderlichkeit der Instanzen der Modell-Klassen korreliert dabei mit der Tatsache, dass auch die Notentexte der zu analysierenden Kompositionen unveränderlich sind. 
,IV-I-V-I-Pendelmodell bezeichnet ${ }^{9}$, Robert Gjerdingen subsumiert sie in seinem Buch Music in the Galant Style unter dem Namen sPrinner (zum Angedenken an den 1624 geborenen und 1694 gestorbenen Musikpädagogen Johann Jacob Prinner)..$^{10}$ Dem Modell folgt ein Halbschluss. ${ }^{11}$ An dieser Stelle sei noch einmal hervorgehoben, dass es üblich ist, transponierte musikalische Wendungen als Instanzen derselben Modellklasse zu interpretieren, weil diese Art der Transformation in der musikalischen Analyse als Operation angesehen wird, welche die wesentlichen Eigenschaften eines Modells nicht berührt.

Die beiden oben abgebildeten Wendungen in B-Dur und in F-Dur entstammen im Original nicht zwei verschiedenen, sondern einer einzigen Komposition, nämlich der Allemande in B-Dur (G 30) von Georg Friedrich Händel (Abb. 3, S. 280). Nach einer Eröffnung durch eine zweitaktige Instanz einer Modell-Klasse, die ich in Anlehnung an eine Arbeit von Warren Kirkendale als $>$ Aria di fiorenza $\iota^{12}$ bezeichne und die bei Gjerdingen unter dem Namen $>$ Romanesca ${ }^{13}$ firmiert (= erster $>A b s a t z \iota$ im Sinne einer paradigmatischen Kadenzfolge nach Heinrich Christoph Koch), erklingt eine zweite - in der Abbildung unbenannte - Taktgruppe in der Subdominante sowie im Anschluss daran das oben abgebildete IV-I-V-I-Pendelmodell mit dem Halbschluss in B-Dur (= zweiter >Absatzı einer paradigmatischen Kadenzfolge nach Koch). Diesen Gestaltungen folgen die Unterquarttranspositionen der zweiten Taktgruppe sowie des IV-I-V-I-Pendelmodells mit Halbschluss (= dritter >Absatz einer paradigmatischen Kadenzfolge im Sinne Kochs). Das hier nicht wiedergegebene Ende des ersten großen Formteils der Allemande bildet ein Ganzschluss in F-Dur (= vierter Gliederungspunkt bzw. Schlusssatz nach Koch). Durch die Tatsache, dass die zwei Exemplare des in Abb. 2 gezeigten Pendelmodells nicht in verschiedenen Kompositionen, sondern in einer einzigen auftreten, wird ein Problem offensichtlich: Obgleich die Modelle im vierten und sechsten Takt bis auf den tonalen Kontext identisch sind, hören wir die erste Passage als Bestätigung der Grundtonart B-Dur $(S \rightarrow T)$, die zweite dagegen als Hinführung zur Dominante $(T \rightarrow D)$ bzw. Modulation in die Tonart der V. Stufe. Die Modelle wirken also trotz gleicher Satztechnik aufgrund der jeweiligen formalen Position und Transposition verschieden. Abb. 3 (S. 280) trägt dem genannten Höreindruck durch eine Erweiterung des Klassendiagramms Rechnung.

9 Kaiser 2007, 166ff. Der Bezeichner einer Modell-Klasse ist ein Name, über den die Klasse eindeutig identifiziert und angesprochen werden kann. Um Missverständnissen vorzubeugen sei darauf hingewiesen, dass ein unsinniger Bezeichner diese Funktion in gleicher Weise erfüllt wie ein semantisch passender, wobei gut gewählte Namen das Verständnis von Programmstrukturen sicherlich erleichtern.

10 Vgl. Gjerdingen 2007, 45 ff. und 455.

11 Zur Bedeutung der Modellkombination `VI-I-V-I-Pendelmodell + Halbschluss für die Gestaltung des Formteils ,Überleitung` in der Musik Mozarts vgl. Kaiser 2007, 211-228, sowie Kaiser 2009.

12 Kirkendale 1972.

13 Gjerdingen 2007, vgl. hierzu $25 \mathrm{ff}$. und 454. 

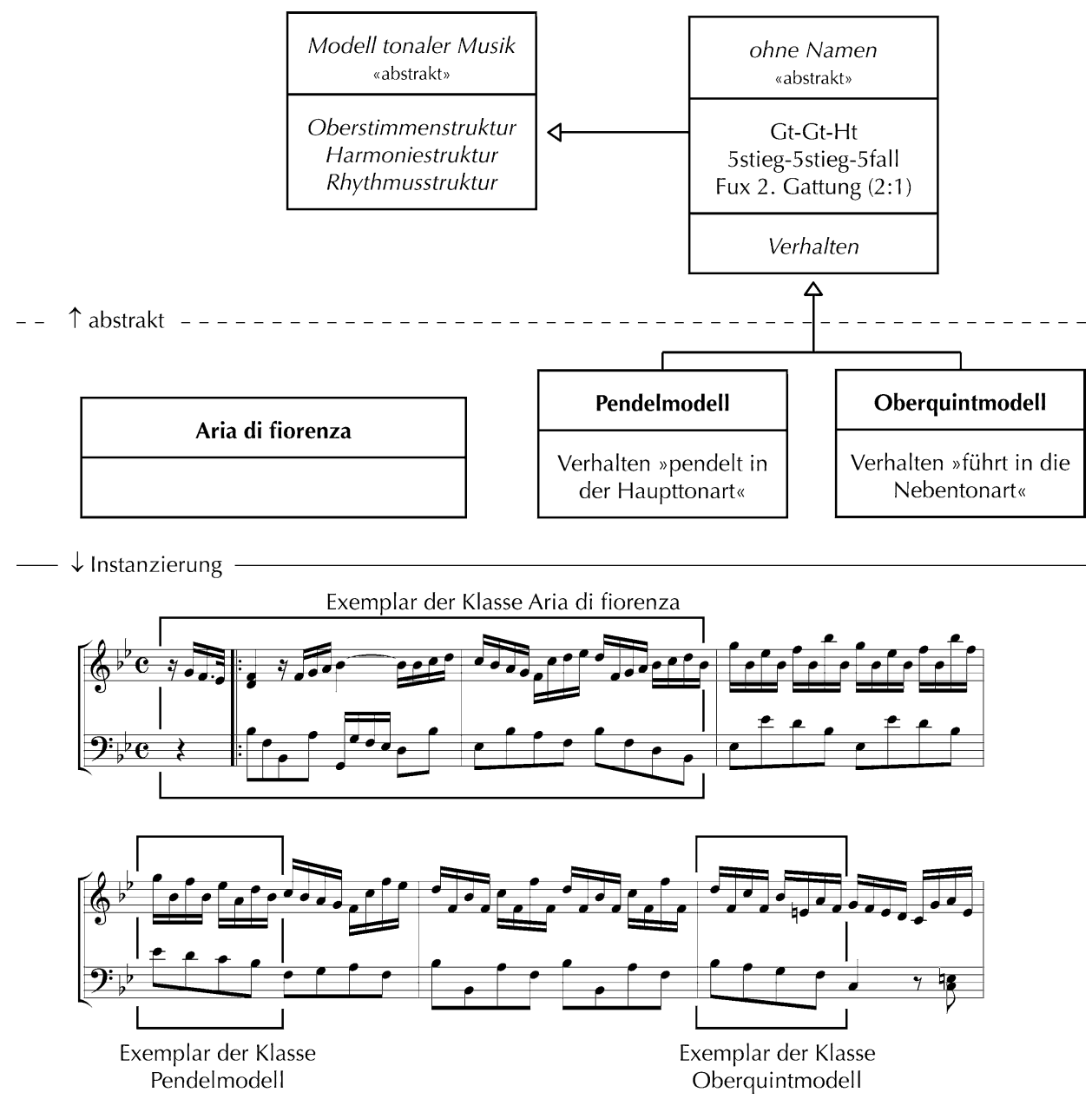

Abb. 3: Georg Friedrich Händel, Allemande in B-Dur (G 30)

Die abstrakte Superklasse >Modell tonaler Musikı deklariert die Eigenschaften Oberstimmen-, Harmonie- und Rhythmusstruktur. Von einer abstrakten Modell-Klasse >ohne Namen werden diese Eigenschaften implementiert und mit spezifischen Werten versehen (Oberstimmenstruktur = Ganzton-Ganzton-Halbton, Harmoniestruktur $=$ QuintstiegQuintstieg-Quintfall sowie Rhythmusstruktur = Fux: 2. Gattung [2:1]). In dieser Form entspricht die abstrakte Subklasse dem Bauplan eines 'Satzmodells` im herkömmlichen Sinne. Darüber hinaus enthält die Klasse sohne Namen s ein abstrakt deklariertes `Verhalten`, das der Beschreibung dient, welche Funktion dem Modell im Kontext einer tonalen Komposition zukommt. Sowohl die bereits konkret implementierten Eigenschaften als auch das abstrakt deklarierte Verhalten der Klasse sohne Namen` werden nun den Sub- 
klassen `Pendelmodelk und `Oberquintmodell vererbt. Die abgeleitete PendelmodellkKlasse erhält durch Vererbung also die konkreten Eigenschaften für Oberstimmen-, Harmonie- und Rhythmusstrukturen ${ }^{14}$ sowie die Verpflichtung zur Implementierung eines spezifischen Verhaltens, das sich z.B. in dem Sinne formulieren ließe, dass von ihr erzeugte Objekte das Gefühl für die Tonart durch ein harmonisches IIV-I-V-I-Pendeln festigen. In der Subklasse `Oberquintmodelk hingegen könnte das Verhalten beschrieben werden als: `Führe in den Bereich der Nebentonart vermittels einer I-V-II\#-V-Harmoniefolge (also das Verhalten eines Modulationsmodells im traditionellen Sinne).

Aus dem Gesagten ergeben sich weitreichende Konsequenzen, und ich finde es interessant, dass der aus der Informatik geborgte Denkansatz der Objektorientierung in der Lage ist, methodisch sauber die Überreste einer positivistischen Musiktheorie zu entsorgen. Denn auf der untersten Ebene der Vererbungshierarchie wurde in Abb. 3 mit

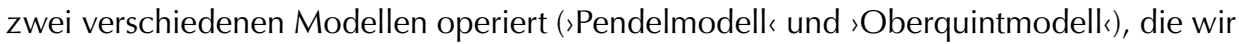
im Rahmen einer herkömmlichen Parameteranalyse (bzw. im Sinne von `Satzmodellen`) als vollkommen identisch bezeichnen müssten.

Der Umstand, dass zwei von einer Superklasse abgeleitete Subklassen ein abstrakt deklariertes und vererbtes Verhalten auf verschiedene Weise implementieren, was zu einem unterschiedlichen Verhalten ihrer Objekte führt, wird in der OOP als >Polymorphie bezeichnet. ${ }^{15}$ Findet dieses Konzept nicht nur auf ein Verhalten, sondern auch auf Eigenschaften Anwendung (z. B. auf die Eigenschaft 〉Harmoniestrukturı), lassen sich als Ergebnis nicht nur aufgrund unterschiedlichen Verhaltens unterschiedliche Modelle mit gleichen Eigenschaften, sondern auch gleiche Modelle aufgrund gleichen Verhaltens mit unterschiedlichen Eigenschaften denken. Beide Fälle sind in der musikalischen Analyse sinnvoll, denn es ermöglicht nicht nur, Modelle bei gleicher Satztechnik aufgrund ihres unterschiedlichen Verhaltens zu unterscheiden, sondern auch zwei musikalische Passagen als gleich zu identifizieren (z. B. als ১Oberquintmodell $\varsigma$, weil sie sich in kompositorischen Kontexten in gleicher Weise verhalten (z. B. von der Grundtonart in die Oberquinte führen), obwohl sie z. B. eine differente Harmonik aufweisen (also in einem positivistischen Sinne ,verschieden sind) ${ }^{16}$

Das bisher entworfene Klassendesign könnte abschließend durch ein wichtiges Konzept moderner objektorientierter Programmiersprachen erweitert werden, nämlich das Konzept sogenannter Schnittstellen. ${ }^{17}$ Schnittstellen dienen der Deklaration von Ver-

14 Dass die Klassen `Pendelmodelk und `Oberquintmodelk die konkreten Eigenschaften GanztonGanzton-Halbton, Quintstieg-Quintstieg-Quintfall sowie 2:1 als Rhythmusstruktur enthalten, lässt sich den jeweiligen graphischen Darstellungen nicht direkt entnehmen, aber anhand der angezeigten Vererbung (durchgezogene Linie mit hohlem Pfeilkopf) erschließen.

15 Die Bezeichnung wird verständlich, wenn man sich vergegenwärtigt, dass Subklassen unter dem Namen der Superklasse angesprochen werden können. Dadurch ist es möglich, dass mit dem Bezeichner einer Superklasse - je nachdem, auf welche Subklasse er verweist - verschiedene Verhalten verbunden sind.

16 Vgl. hierzu das Kapitel `Oberquintmodelle` in Kaiser 2007, 211-228.

17 Schnittstellen sind spezielle Klassen, die Definitionen von Eigenschaften, Methoden und Ereignissen enthalten dürfen, nicht jedoch deren Implementierungen (also z. B. nicht die konkrete Ausformulierung eines Eigenschaftswertes). In dieser Hinsicht gleichen Schnittstellen den abstrakten Klassen: Beide sind unselbständig und bedürfen der Einbindung durch weitere Klassen, die den entspre- 
einbarungen, an die sich alle Klassen halten müssen, welche die jeweilige Schnittstelle implementieren. Eine Schnittstelle beispielsweise, von der die Eigenschaft shat Saiten definiert und die von der Klasse Streichinstrumente implementiert wird, verpflichtet alle Streichinstrumente dazu, Saiten zu haben. Die gleiche Schnittstelle kann zudem ıVertragspartner verschiedener Klassen sein. Es wäre z. B. sinnvoll, wenn auch die Klasse Zupfinstrumente eine vertragliche Verpflichtung mit der Schnittstelle shat Saiten hen und somit Zupfinstrumente verpflichten würde, Saiten zu haben. Das Auslagern von shat Saiten « wird dabei in der Objektorientierung als `Kapselung` bezeichnet. Der Vorteil gekapselter Eigenschaften liegt darin, dass diese von den implementierenden Klassen unabhängig gedacht werden können: z. B. ist die Eigenschaft shat Saiten unabhängig von den Klassen `Streichinstrumente` und ¿Zupfinstrumente stelle) auch für eine Klasse `Klavierinstrumente` zur Verfügung. In Bezug auf das bisher entworfene Modell-Klassendesign wäre es daher möglich, die in der abstrakten Klasse sohne Namen` implementierte Oberstimmenstruktur `Ganzton-Ganzton-Halbton`, die Harmoniestruktur `Quintstieg-Quintstieg-Quintfalk sowie die Rhythmusstruktur `Fux: 2. Gattung $(2: 1)$ < zu kapseln bzw. in Schnittstellen auszulagern. Der Vorteil läge in der Kombinierbarkeit (und hinsichtlich der Programmierung in der Wiederverwendbarkeit) der genannten Eigenschaften für alle erdenklichen Modell-Klassen tonaler Musik (Abb. 4).

Bevor ich auf die Art des Instanzierens in einer musikalischen Analyse eingehen und verschiedene Probleme ansprechen werde, seien zuvor noch einige klärende Überlegungen zum Begriff des Verhaltens gestattet. Wie bereits erwähnt, ist unter dem Verhalten eines Modells seine spezifische Funktion innerhalb einer bestimmten Komposition zu verstehen. Während sich Eigenschaften also direkt an einer Modellinstanz beobachten lassen, ist eine Bestimmung des Verhaltens nur über den kompositorischen Kontext möglich. Die denkbaren Funktionen eines Modells in einer Komposition fallen dabei weder mit den harmonischen Funktionen 'Tonikas, 'Subdominante

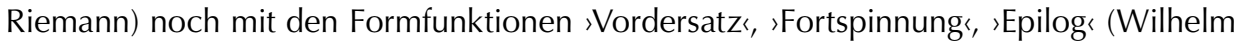
Fischer) bzw. `Presentation`, ‘Continuation` und `Cadential (William Caplin) zusammen. Das Denken in den elementaren Formfunktionen `Anfang`, 'Mitte erst von Fischer und Caplin beschrieben worden ist, sondern bereits in der Improvisations- und Kompositionsdidaktik des 18. Jahrhunderts Konjunktur hatte ${ }^{18}$, stellt zwar für

chenden Code bereitstellen. Das Konzept der Schnittstellen wurde eingeführt, weil in modernen objektorientierten Programmiersprachen die sehr fehleranfällige Mehrfachvererbung ausgeschlossen worden ist und man es dennoch für wünschenswert hielt, neben einer Basisklasse weitere übergeordnete Kategorien (Schnittstellen) zuzulassen, die der Beschreibung gemeinsamer Merkmale vieler ansonsten unabhängiger Klassen dienen.

18 Im `Fundamentum del Eberlin<, einer in der Bayerischen Staatsbibliothek befindlichen Handschrift (Mbs Mus. Ms. 261), die als Abschrift einer rars componendis des Salzburger Hoforganisten und Hofkapellmeisters Johann Ernst Eberlin (1702-1762) gilt, heißt es, dass »das ganze Praeambulieren in nichts anderen bestehe, als daß ich erstens weis anzuschlagen, darnach und zweytens etliche Sekunden, 3ten, quarten etc. von einem ton in den andern zu spillen und endlich drittens ein Final zu machen (Praefatio ad Discipulum). Ideengeschichtlich lässt sich ein Denken in den Formfunktionen 'Anfang`, 'Mitte Anfänge der Kompositionslehren musikalischer Rhetorik: "Resulutio Cantilenae in affectiones, est diviso cantilenae in periodos, ad disquirendum artificium, \& idipsum ad imitatione convertendum. Haec tres habet partes, 1. Exordium, 2. Ipsum corpus carminis, 3. Fines« (Burmeister 1606, 72). 


\begin{tabular}{|c|}
\hline $\begin{array}{c}\text { Gt-Gt-Ht-Modell } \\
\text { «Schnittstelle» }\end{array}$ \\
\hline $\begin{array}{c}\text { Oberstimmenstruktur } \\
\text { Gt-Gt-Ht }\end{array}$ \\
\hline
\end{tabular}

4

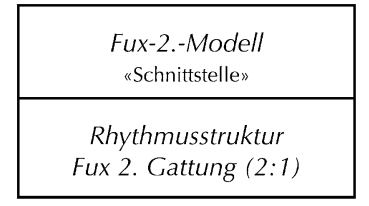

Fux 2. Gattung $(2: 1)$
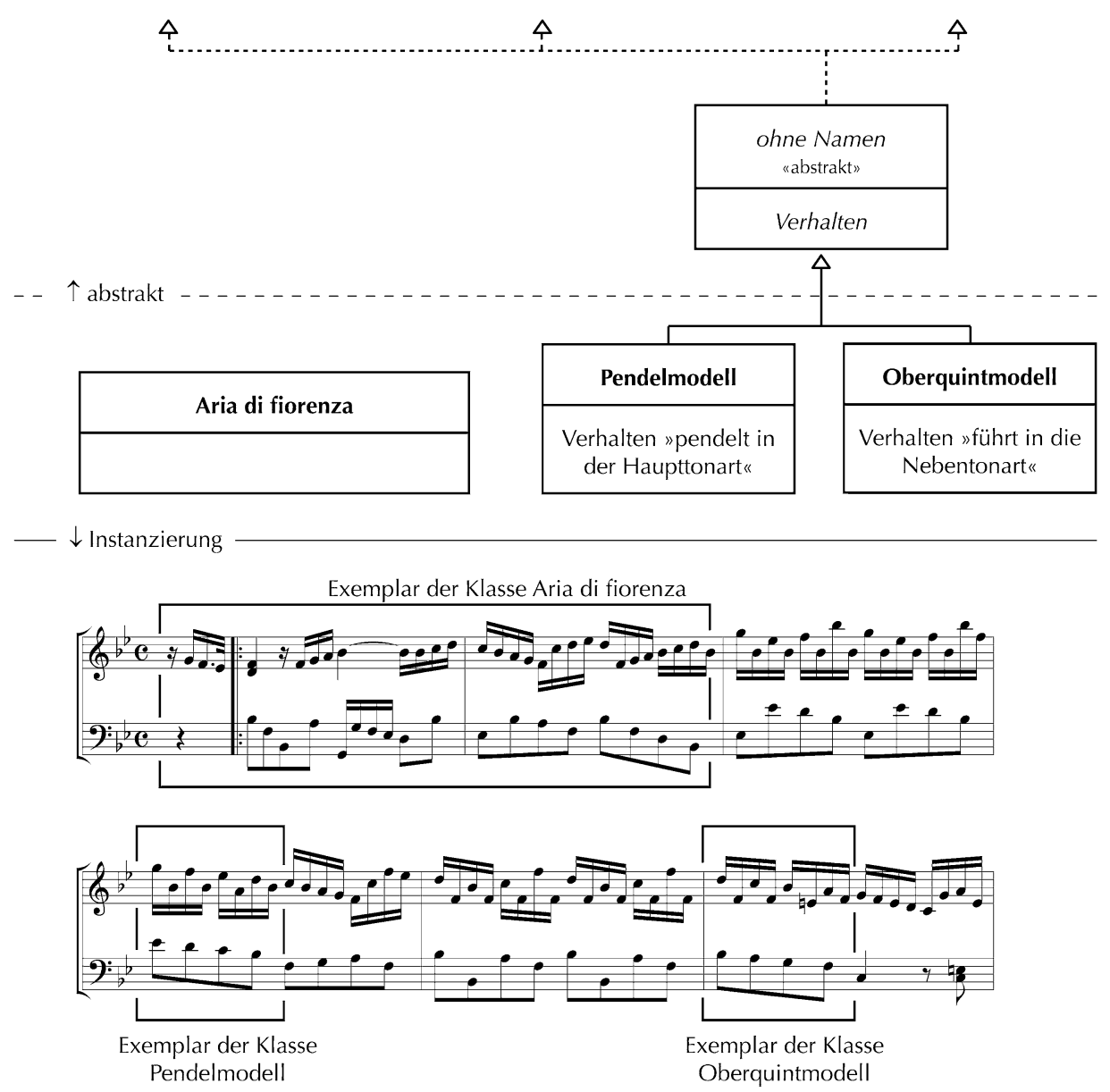

Abb. 4: Kapseln konkreter Eigenschaften mit Hilfe von Schnittstellen

die Analyse nützliche Verhalten bereit, die Beschränkung auf eine bestimmte Anzahl von Formfunktionen bzw. Verhaltensweisen wäre jedoch dogmatisch und speziellen Interessen verpflichtet. In meiner Arbeit Die Notenbücher der Mozarts habe ich dem `Verhalten` (bzw. der Formfunktion) darüber hinaus die Kategorien ıInszenierungsweise` und >Ausdehnung a an die Seite gestellt (im Sinne abstrakter Superklassen-Deklarationen). Mit der Verpflichtung, die Ausdehnung eines Modells hinsichtlich seines Kontextes zu 
beschreiben, lässt sich vergegenwärtigen, dass in der Analyse üblicher Weise nicht konkrete musikalische Erscheinungen, sondern deren musiktheoretische Abstraktionen verglichen werden. Die Aussagefähigkeit der Gleichheit von I-V-I-Harmoniefolgen ist daher gering, wenn einmal eine Kadenzwendung, ein anderes Mal eine Taktgruppenharmonik und ein drittes Mal ein Satzverlauf beschrieben wird. Über die Kategorie ıInszenierungsweise lassen sich zudem standardisierte musikalische Gesten wie z. B. ein rauschendes Orchestertutti als Abschluss eines größeren Formteils oder ein bassloses Register mit solistischen Bläserfarben an der Position eines Seitensatzes oder Seitenthemas benennen, wobei spezifische Inszenierungsweisen die Wirkung gleicher Satztechniken so grundlegend verändern können, dass es sinnvoll erscheint, in solchen Fällen von unterschiedlichen Modellen zu sprechen.

Die Akte der Instanzierung, nicht jedoch die Vorgänge des Instanzierens lassen sich in der Computerwelt und im Rahmen einer musikalischen Analyse vergleichen. Während das Konzept der Objektorientierung in der Computerwelt dadurch aufgelöst wird, dass eine triviale Maschine ${ }^{19}$ (Compiler) einen ausführbaren Maschinencode erzeugt, der eine Äquivalenz zum modellierten Programmdesign darstellt, erfolgt das Instanzieren von Modellen im musiktheoretischen Kontext in Form von Beobachtungen an einer Komposition durch Nicht-Trivialmaschinen (Menschen). In Anlehnung an einen »extrem formalen Begriff des Beobachtens $\aleph^{20}$ wird hier unter Beobachtung das Benutzen einer Unterscheidung sowie das Bezeichnen des Unterschiedenen verstanden. In der Allemande von Händel beispielsweise wurden die ersten beiden Takte vom Rest der Komposition unterschieden und für ihre Bezeichnung der Begriff >Aria di fiorenza` gewählt. Die Operation des Beobachtens (bzw. Unterscheidens und Bezeichnens) fällt dabei mit dem Akt der Instanzierung zusammen, das durch den Begriff Bezeichnete repräsentiert die Instanz einer entsprechenden Modell-Klasse.

Beobachtungen dieser Art sind stark wissensgeleitet und widersprechen dem gelegentlich anzutreffenden Anspruch, die Art des Beobachtens in der musikalischen Analyse müsse »möglichst unvoreingenommen « erfolgen. ${ }^{21}$ Diese Forderung dürfte dem Bemühen geschuldet sein, Beschreibungen nicht von vornherein musiktheoretischer Dogmatik auszuliefern. Doch der Versuch, Musik so beobachten zu wollen, wie sie ist (und nicht so, wie sie nicht ist), setzt die ontologische Sein-Nichtsein-Unterscheidung voraus und zwingt zu einem Realitätsverständnis aus der Perspektive des Subjekt-Objekt-Schemas. Abgesehen von den erkenntnistheoretischen Problemen, die hierbei zwangsläufig mitgeschleppt werden, verschleiert die Forderung nach Unvoreingenommenheit den Widerspruch zwischen der Freiheit, die hinsichtlich der zahlreichen Beschreibungsmöglichkeiten eines Gegenstandes nach Aufhebung der Limitation durch Voreingenommenheit existiert, und den Zwängen der Kausalität, die mit Kommunikationsabsichten, Unter-

19 Unter einer Trivialmaschine wird eine Transformationsfunktion verstanden, die nach starren Regeln einen Input in einen Output verwandeln (z. B. ein Klavier, ein Toaster etc.). Nicht-Trivialmaschinen dagegen reagieren selbstbestimmt, d. h. durch Ermittlung einer Selbstreferenz in Bezug auf die Verarbeitung des Inputs, was für Außenstehende den Output nicht vorhersehbar macht.

20 Die folgenden Ausführungen sowie die Wahl der Terminologie sind beeinflusst von der Lektüre ausgewählter Schriften Niklas Luhmanns (insbesondere einiger Kapitel aus Luhmann 1990).

21 Vgl. hierzu z. B. Küster 2001, 39-45. 
richts- und Lernzielen einhergehen. Die Forderung nach Unvoreingenommenheit dürfte also schon allein deshalb scheitern, weil das Beobachten der Forderung nach Unvoreingenommenheit selbst Voreingenommenheit erzeugt.

Wird die Forderung nach einer möglichst unvoreingenommenen Beschreibung zurückgewiesen, bedeutet dies zugleich einen Versuch preiszugeben, den Einfluss musiktheoretischer Dogmatik im Rahmen einer musikalischen Analyse zu kontrollieren. Es bleibt daher die Frage, auf welche Art sich eine musikalische Analyse, die den Anspruch auf Wissenschaftlichkeit erhebt, vom kultischen Gebrauch einer analytischen Methode unterscheiden lässt, zumal auch für wissenschaftliche Fragestellungen Begrenzungen notwendig sind, ohne die sich jede Untersuchung ins Unendliche verlieren würde. Als Antwort sei auf Luhmanns Unterscheidung zwischen `Theorie sen. Nach dieser Unterscheidung sind Methoden `Programme`, die einem binären Code verpflichtet sind und kein anderes Ziel haben als "eine Entscheidung zwischen wahr und unwahr herbeizuführen «. ${ }^{22}$ Diese Programme befinden sich in strikter Koppelung mit Theorien bzw. anders gearteten `Programmen`, die spezifizieren, unter welchen Bedingungen die Qualifikation ıwahr oder sunwahr Codierung nicht unterworfen sind, bestehen aus Aussagen in Form von Sätzen, die sich auf etwas anderes als sich selbst beziehen (z. B. auf einen Untersuchungsgegenstand, Luhmann spricht in diesem Zusammenhang von sassoziierter Fremdreferenz bzw. einer seingebauten Asymmetrie «). ${ }^{23}$ Das Zusammenspiel von Theorie und Methode wird sehr pragmatisch erläutert:

Beide Arten von Programmen können unter wie immer willkürlichen und vorläufigen Limitierungen in Operation gesetzt werden, da jede Limitation von der anderen Seite der Unterscheidung her infrage gestellt und gegebenenfalls ausgewechselt werden kann. Limitation ohne Limitation also! Die Theorien können ausgewechselt werden, je nachdem, was ihre methodische Überprüfung ergibt. Und die Methoden werden gewählt, korrigiert und gegebenenfalls weiterentwickelt je nach dem, was man zur Überprüfung von Theorien braucht $\left[\ldots . .{ }^{24}\right.$

Um Missverständnissen vorzubeugen sei erwähnt, dass Luhmann mit der Formulierung „wie immer willkürlich « ${ }^{25}$ kein svollkommen beliebiges` In-Operation-Setzen von Theorie und Methode meint:

Man formuliert nicht aufs Geratewohl Sätze, um dann sich um die Feststellung ihrer Unwahrheit zu bemühen. Die festzustellende Unwahrheit muß sinteressant sein, muß also im Falle ihrer Wahrheit eine sinnvolle Theorie ergeben. Andernfalls wäre alles möglich und das System hätte an seiner Struktur nicht genügend Führung [...]. ${ }^{26}$

22 Luhmann 1990, 415.

23 Ebd., 406.

24 Ebd., $403 \mathrm{f}$.

25 »Wie immer besagt 'willkürlich` auch hier: beobachte den Beobachter! « (ebd., 408, Fn. 66)

26 Ebd., 205. 
Daher wird für den Beginn eines Theorieaufbaus empfohlen:

Sicher ist die einfachste Auskunft eine rein historische: Man arbeitet mit den Unterscheidungen, die sich schon bewährt haben und deren Kondensate die Formulierungen von Theorien ermöglichen, die ihrerseits Erkenntnisse festhalten, die man für wahr hält. ${ }^{27}$

In Anknüpfung an das theoretische Werk Joseph Riepels und daran orientierten Forschungsarbeiten zeigt Abb. 5 ein exemplarisches In-Gang-Setzen von Theorie und Methode im Bereich musikalischer Analyse. Damit dürfte niemandem, der heute dieses Geschäft ernsthaft betreibt, wirklich etwas Neues gesagt sein. Doch hinsichtlich eines noch zu etablierenden wissenschaftlichen Fachs, bei dem das Wort 'Theorie im Namen so ernst genommen wird, dass Musiktheorie nicht nur als historische, sondern auch systematische Disziplin verstanden wird, könnte es von Bedeutung sein zu vergegenwärtigen, was aus der dargelegten Perspektive die Kriterien der Wissenschaftlichkeit nicht erfüllt: Das Bestimmen von Modellinstanzen, Chiffrieren von Akkordfunktionen, Benennen von Stufenfolgen, Konstruieren von Stimmführungszügen, Operieren mit Tonfeldern etc. - es sei denn, das bisher bekannte methodische Arsenal der Musiktheorie wäre in den Dienst der Qualifizierung (wahr/unwahr) theoretischer Sätze gestellt (z. B. in der Form: "spezifische Expositionen des Komponisten $\mathrm{x}$ aus den Jahren y lassen sich als Ausprägung der Modellkombination z verstehen « oder allgemeiner »Musik ab x lässt sich angemessen als spezifische Abfolge y der Tonfelder z beschreiben« etc.). Selbst die kapriziöse Beherrschung analytischer Methoden wäre demnach Propädeutik (in einem positiven Wortsinn), das Operieren mit theoretischen Sätzen ohne methodische Überprüfbarkeit $^{28}$ (z. B. das Konstatieren von Komponistenintentionen) allenfalls Bestandteil eines didaktischen Designs zur Erweckung von Motivation (im Unterricht). Spezifisch wissenschaftliche Leistungen wären letztendlich daran zu bemessen, ob es gelingt, die Abstraktion der Vergleichsgesichtspunkte so weit voranzutreiben, dass »auch evident Ungleiches verglichen werden kann (und nicht nur der Erfüllungsgrad von Wünschen gemessen wird).$^{29}$

Abschließend sei die Frage nach der Bedeutung des zu Beginn dargelegten ModellKonzepts im Rahmen einer wissenschaftlichen musikalischen Analyse gestattet. Das Instanzieren (bzw. Beobachten) eines Modells ist eine beobachtbare Operation, das Vergegenwärtigen einer Modellklasse im objektorientierten Sinn Resultat dessen, was von Luhmann als Wechsel des Beobachters oder als Anweisung »an eine Beobachtung

27 Ebd., 379.

28 »Gäbe es als Selektionskriterium nur die vorhandenen Theorien, liefe das auf eine Abweisung aller Variation hinaus. Die bereits etablierten Theorien wären das Kriterium für ihren eigenen Fortbestand. Das richtige Wissen könnte zwar Abweichungen erkennen, könnte sich selbst aber nicht in Frage stellen. Erst in dem Maße, als zusätzlich zu Theorien auch Methoden Programme für richtige Selektionen werden (und zwar spezialisiert nicht auf Weltbeschreibung, sondern auf die Probleme der binären Codierung), erhält die Selektion sozusagen ein zweites Bein, mit dem sie einen anderen Standplatz suchen kann.«(Ebd., 578f.)

29 Ebd., $409 \mathrm{f}$. 


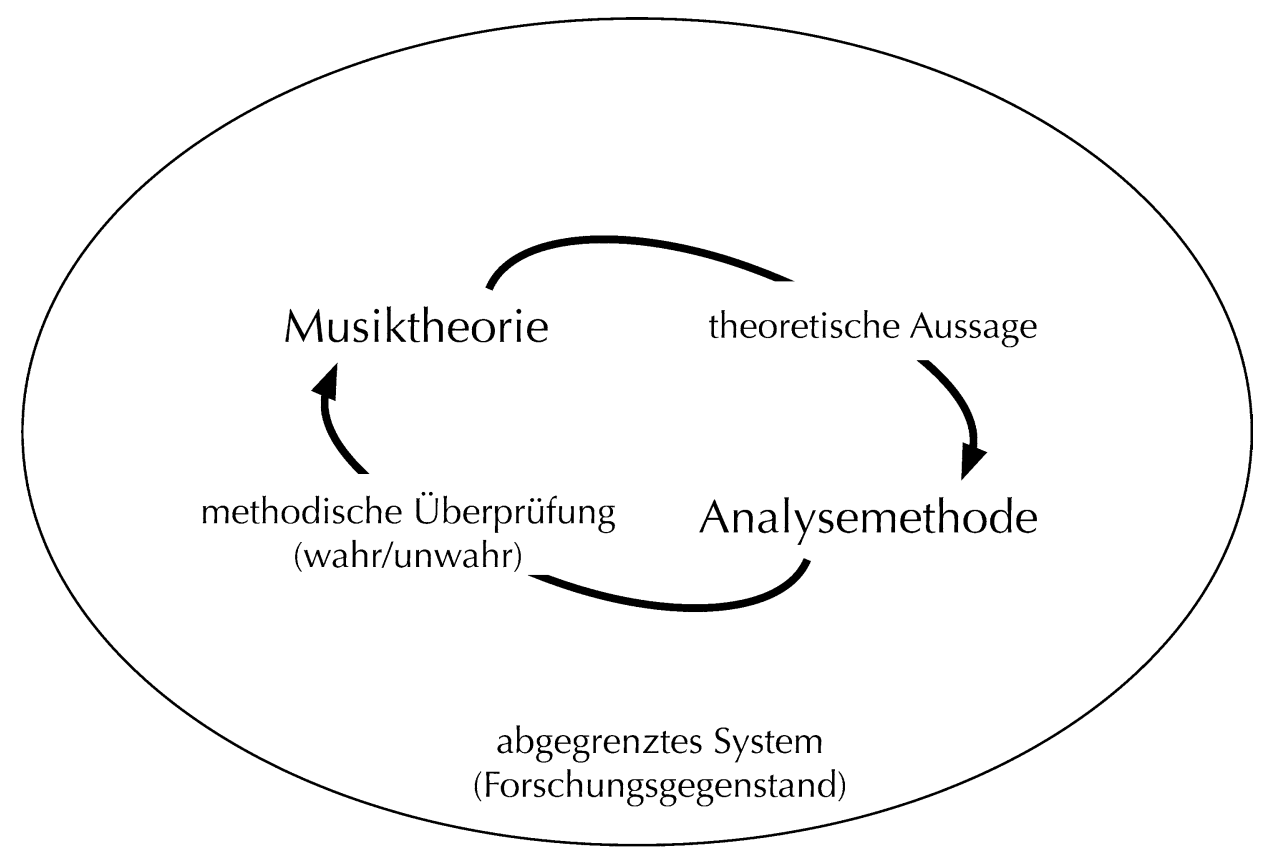

Theorie Durchführungen in Mozarts frühen Kompositionen lassen sich als Zusammensetzung aus Fonte-, Monte- oder PonteModellen verstehen.

Methode Unwahr. Eine Untersuchung mit Hilfe von Modellinstanzen ergibt, dass Mozarts Durchführungen in dieser Zeit häufig mit einem Kadenzmodell »Halbschluss« enden.

Theorie Durchführungen in Mozarts frühen Kompositionen zeigen Zusammensetzungen aus Fonte-, Monte-, Ponte-Modellen sowie einem Halbschluss.

Methode Unwahr. Z. B. lässt sich die Durchführung aus KV 6/IV nicht mit Hilfe dieser Modell-Klassen beschreiben.

Theorie Es gibt spezifische Durchführungen in Mozarts frühen Kompositionen, die sich als wechselnde Kombinationen folgender Modelle beschreiben lassen: Fonte-, Monte-, Ponteund Halbschluss.

Methode

Wahr. Z.B. KV 6/I, KV 6/II, KV 7/I, KV 10/II KV 14/I etc.

Abb. 5: Wissenschaft als strikte Koppelung der Programme `Theorie` und `Methode` 
von Beobachtern « bezeichnet worden ist. ${ }^{30}$ Nur die Reflexion dessen, was als Modell instanziert wurde (und warum), garantiert die Möglichkeit der gezielten Entwicklung neuer Modelle. Die Rhythmusstruktur >Fux 2. Gattung (2:1)< beispielsweise wäre als konstitutive Eigenschaft für Pendel- und Oberquintmodelle wenig geeignet, denn sie ermöglicht bestenfalls eine Bewahrheitung theoretischer Aussagen zu einer spezifischen musikalischen Stilistik aus der ersten Hälfte des 18. Jahrhunderts. Wird diese Eigenschaft jedoch vernachlässigt, qualifiziert sich das Modell auch für die Beschreibung der Musik Mozarts bzw. zur Bewahrheitung von Aussagen über nicht stilgebundene Entwicklungszüge tonaler Musik (z.B. Aussagen zur Entwicklung der Sonatenhauptsatzform, wofür im Übrigen auch die nach Koch stimmige Absatzdisposition als Indiz gewertet werden könnte). Die Verpflichtung zur Einhaltung des beständigen Perspektivwechsels (zwischen Beobachtungen erster und zweiter Ordnung) ist unabdingbare Voraussetzung für die Ausbildung einer Methodologie und Entwicklung der Analyse nach Modellen jenseits von sParallelismus, Quintfallsequenz \& Co . Nicht zuletzt wäre ein solcherart differenziertes Denken von Modellen geeignet, wenig ergiebige Fachgespräche zu vermeiden, weil sich schnell vergegenwärtigen ließe, ob beim Verwenden gleicher Begriffe gleiche Modelle benannt werden und ob Theorieprogramme unterschieden (oder gar nicht vorhanden) sind, wodurch sich wiederum eine Diskussion über die Unterschiedlichkeit (oder Beliebigkeit) von Modellen erübrigt.

Über die eingangs gestellte Frage `Was ist ein musikalisches Modell? « wurde ein an der Objektorientierung angelehntes Denken musikalischer Modelle für tonale Musik erläutert sowie der Versuch unternommen, dieses Denken in einem an Luhmanns Ausführungen orientierten Wissenschaftskonzept zu verorten. Damit dürften allerdings mehr Fragen aufgeworfen denn beantwortet sein, z. B.: Wie müssten Analysemethoden für eine Musik beschaffen sein, die nicht unter das ästhetische Paradigma einer sars combinatoria (im weitesten Sinne) fällt und die sich gegen das hier dargelegte Analysieren nach Modellen sperrt? Welche Arten theoretischer Sätze sind über eine Musik möglich, die einen Komplexitätsgrad aufweist, der viele musikalische Erscheinungen des 20. und 21. Jahrhunderts kennzeichnet? Und auf welche Weise müssen theoretische Sätze und Methoden hinsichtlich einer Musik vorgestellt werden, deren Beschreibung sich traditioneller Analyse am Notentext weitgehend entzieht (z. B. hinsichtlich einer mit Hilfe von hochkomplexer Studiotechnik gefertigten Populären Musik)? Um den hier vorgestellte Ansprüchen an wissenschaftliches Arbeiten genügen zu können, dürfte das Entwickeln theoretischer Fragestellungen sowie geeigneter Analysemethoden zu den größten Herausforderungen für Fachvertreterinnen und Fachvertretern gehören, die den systematischen Aspekt des Fachs Musiktheorie nicht einer einseitig historischen Betrachtungsweise zu opfern gewillt sind. 


\section{Literatur}

Anon., Modus brevissimus desumpta [!] ex Regulis D: Eberle [!] Salisburgis de arte Componendi, D-Mbs Mus. ms. 261.

Burmeister, Joachim (1606), Musica poetica, Rostock, Reprint Laaber: Laaber 2004.

Fuß, Hans-Ulrich, (2007), »Subthematische Arbeitı. Komponieren mit mehrstimmigen Satzmodellen bei Mozart«, ZGMTH 4/1-2, 87-106.

Gjerdingen, Robert O. (2007), Music in the Galant Style, New York: Oxford University Press.

Kaiser, Ulrich (2007), Die Notenbücher der Mozarts als Gundlage der Analyse von W. A. Mozarts Kompositionen 1761-1767, Kassel: Bärenreiter.

- (2009), „Der Begriff der ,Überleitung` und die Musik Mozarts. Ein Beitrag zur Theorie der Sonatenhauptsatzform«, erweiterte Schriftfassung eines Referats auf der 6th European Analysis Conference in Verbindung mit dem VII. Jahreskongress der Gesellschaft für Musiktheorie 2007 in Freiburg, ZCMTH 6/2-3.

Kirkendale, Warren (1972), L'Aria di Fiorenza id est Il Ballo del Gran Duca, Florenz: Olschki.

Küster, Konrad (2001), W. A. Mozart und seine Zeit (= Große Komponisten und ihre Zeit), Laaber: Laaber.

Luhmann, Niklas (1990), Die Wissenschaft der Gesellschaft, Frankfurt a. M.: Suhrkamp. Schwab-Felisch, Oliver / Hans-Ulrich Fuß (2007), »Editorial«, ZGMTH 4/1-2, 9-12. 